\title{
LEHRBUCH
}

DER

\section{DARSTIELLENDEN GEOMETRIE}

VON

Dr. KARL ROHN

O. PROFESSOR DER MATHEMATIK

$\triangle N$ DER UNIVERSITÄT LEIPZIQ
UND DR. ERWIN PAPPERITZ

O. PROFESSOR DER MATHEMATIK AN DER BEREAKADEMIE FREIBERO

DRITTE, UMGEARBEITETE AUFLAGE IN DREI BÄNDEN

ZW EITER BAND

AXONOMETRIE, PERSPEKTIVE, BELEUCHTUNG.

MIT 118 FIGUREN IM TEXT.

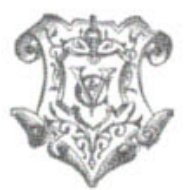

LEIPZIG

VERLAG VON VEIT \& COMP. 
Druck von Metzger \& Wittig in Leipzig. 